Section name: 02ii. Classical Greek philosophy

Markus Riedenauer

\title{
Platon: Kein Staat
}

ABSTRACT: Eines der einflussreichsten Bücher der westlichen Philosophie ist die Politeia von Platon - meist mit „Der Staat“ übersetzt. Leicht ist erkennbar, dass das Werk viel mehr als politische Philosophie enthält - der Beitrag fasst insgesamt zwölf Argumente dafür zusammen, dass es überhaupt keine überzeugende politische Philosophie enthält. Die erkenntnistheoretischen, metaphysischen und anthropologischen Voraussetzungen und Argumente der Politeia erlauben keine adäquate Beschreibung und Reflexion der Natur eines Staates, noch seines Bezugs zu den Individuen, die ihn konstituieren, noch des Charakters politischer Institutionen oder der politischen Sphäre an sich.

Selbst wenn angenommen wird, dass die erste Absicht des Werks gewesen sei, Gerechtigkeit und Moralität auf individualethischer Ebene zu begründen, verhindern die idealistischen und dualistischen Grundelemente ein Verständnis von Tugenden und Handlungsnormen.

Erst recht ist es unmöglich, den Staat aufgrund der Prämissen der Politeia selbst zu konstruieren oder theoretisch zu rekonstruieren. Das Scheitern Platons als politischer Berater verdankt sich nicht oder nicht nur kontingenten historischen Faktoren, sondern vor allem den Defiziten seiner politischen Theorie.

SCHLÜSSELWÖRTER: Plato, Politeia, state, justice, ethics, idealism

Wozu schrieb Platon die Politeia? Gemäß 368d-369a ausdrücklich mit ethischer Zielsetzung, damit nämlich am großen Bild leichter ablesbar würde, was (individuelle) Gerechtigkeit ist. Daraus ließe sich schließen, dass nach der ersten Intention des Autors keine politische Philosophie zu erwarten ist ${ }^{1}$.

Das ist aber als Interpretationsansatz des ganzen Werks unbefriedigend. Denn es geht weit über die Parallelen von Einzelseele und Polis hinaus, v.a. bei Fragen der Reproduktion und Erziehung im Staat, für die es kein Äquivalent im Individuum gibt. Außerdem bedingen sich die Entwicklung eines individuellen Ethos durch Erziehung und die Gestaltung der pädagogischen und anderen Rahmenbedingungen gegenseitig. Internalisierung und Externalisierung von Normen bilden generationenübergreifend ein Bedingungsgefüge, das eine gute Ordnung des Staates für die

\footnotetext{
${ }^{1}$ Demnach würde die Kritik Karl Poppers auf einer Fehldeutung beruhen - allerdings ist er sich bewusst, der Ansatz sei ,an analysis of the individual rather than that of the state" (1963, S. 79, cf. 89). Dagegen argumentiert Schweidler, dass Platon einen „viel bescheideneren Anspruch“ habe als Sophokratie und Führerstaat zu rechtfertigen, er reklamiere „lediglich, dass das politische Engagement des Philosophen nicht völlig irrational sei“ (2004, S. 35). Wenn das alles wäre, dann wäre die Politeia weniger gefährlich als Popper annahm, weil sie gar keine politische Philosophie zu entfalten beansprucht. Die im Folgenden zusammengestellten Argumente zeigen, dass sie es jedenfalls nicht zu Recht beanspruchen kann.
} 
Gerechtigkeit des Individuums erfordert - andernfalls werden Gerechte wie Sokrates letztlich eliminiert ${ }^{2}$.

Inwieweit wollte Platon also doch politische Philosophie betreiben? Nach dem Zeugnis des 7. Briefes, der Nomoi und des Politikos hegte Platon die Überzeugung, dass Gesellschaft und Staat erst gesunden, wenn die Weisheit herrscht - und dies sei nur möglich in Form der Herrschaft ihrer Liebhaber, der Philosophen ${ }^{3}$. Von diesen beiden Thesen ist die erste trivial, wenn sie die Notwendigkeit einer vernünftigen Ordnung überhaupt meint, und die zweite tendenziell totalitär ${ }^{4}$. Die leichthändige Verknüpfung beider (vor allem im Philosophenkönigssatz der Politeia) verdankt sich problematischen anthropologischen und metaphysischen Vorentscheidungen. Die Tendenz zur Umsetzung des Programms zeigt sich auch darin, dass Platon in Syrakus und andernorts versuchte ${ }^{5}$, seine Ideen in die Praxis umzusetzen (auch wenn dies natürlich nicht gleich bedeutet, er habe einfach die Politeia realisieren wollen).

Bekanntlich gelang ihm das nicht. Scheitern kann immer verschiedene kontingente und praktische Ursachen haben, aber meine These ist, dass dahinter fundamentale theoretische Defizite stehen. Nicht erst aufgrund einer Konfrontation mit modernem politischen Denken wie bei Popper, sondern schon werkimmanent ist festzustellen: Mit Platons Denken ist kein Staat zu machen. Dafür fasse ich sieben Argumente zusammen, manche davon mit Unterargumenten.

\section{Mangelndes Verständnis des Sozialen und Politischen an sich:}

1a. Ethik und Politik sind für Platon so sehr eins, dass die Eigenart des Staates als Vereinigung von Individuen und kleineren Gemeinschaften (vor allem Familien) und damit die Möglichkeit je unterschiedlicher moralischer Bedingungen und Kriterien übersehen wird. Die fatale Folge ist, dass Platon dem Staat eine Einheitsvorstellung wie von einem Menschen unterstellt ${ }^{6}$ und die Eigengesetzlichkeit der politischen Sphäre verdeckt wird.

1b. Das Einheitsideal ist aber wiederum nicht aus der Wirklichkeit menschlichen Zusammenlebens erhoben, sondern als abstrakte Idee postuliert und dann von außen bzw. oben oktroyiert. Die Einheit eines (menschlichen) Organismus ist in Wahrheit eine ganz andere, von innen

\footnotetext{
${ }^{2}$ So weit ist Thurnher (2012) Recht zu geben, dass Platons Staatsutopie zeigen will, in welcher Polis der Philosoph eine Heimat finden könnte. Jedoch könnte sich ein Philosoph schwerlich seines Menschseins so entledigen, dass er in einem rein fiktiven Staat als reiner Geist leben könnte - die Tendenz zur Realisierung ist unausweichlich und der 7. Brief gibt davon Zeugnis (siehe 328c).

${ }^{3}$ Pol. V 473d; vgl. 7. Brief 324c-326b, 328a. Das Urbild ist der Sokrates des Gorgias (521d), der glaubt, praktisch als einziger in Athen die wahre Staatskunst zu pflegen.

${ }^{4}$ Gewalt oder Freiwilligkeit, Gesetz oder Gesetzlosigkeit sei kein Kriterium für die Qualität einer Verfassung, königliche Herrschaft wird als Wissenschaft charakterisiert, vgl. Politikos 292a-293e.

${ }^{5}$ Siehe 7. Brief und (vermutlich unechte) andere Briefe, v.a. 5. und 6.; vgl. v. Fritz: „der Verlauf des Geschehens gerade in diesem exemplarischen Falle läßt durch alle positiven und negativen Zufälligkeiten hindurch besonders deutlich erkennen, warum entgegen der Meinung Platons der Versuch des ,Philosophen', sich aktiv an der Politik zu beteiligen, notwendig scheitern muß“ (1968, S. IX). Dafür bringt v. Fritz andere Argumente als ich, weil er die Probleme nicht aus Platons Theorie, sondern vor allem aus der Natur des Menschen und der Politik entstehen sieht.

${ }^{6}$ Siehe Politeia V 462c. Aristoteles erkannte darin das metaphysische Grundproblem, während er sonst in seiner Kritik des platonischen Staats einen realistischen Ansatz verfolgt, der die Vorschläge vor allem zum radikalen Kommunismus wörtlich nimmt und von ihren Folgen her kritisiert (Aristoteles: Politika II,1-5 1260b-1264b).
} 
und von selbst sich ständig bildend, als die durch Repression der Wissenden bewirkte Einheit oder Pseudo-Einheit des Staatsentwurfs ${ }^{7}$. Pluralität widerspricht diesem abstrakten Einheitsbegriff a priori.

1c. Politische Institutionen, wie in der Verfassungslehre der Politeia VIII-IX skizziert, sind rein eine Folge des Seelenzustands von Individuen oder einer Menge von (aristokratischen, timokratischen, oligarchischen, demokratischen, tyrannischen) Individuen. Eine soziologisch beschreibbare Eigenwirklichkeit und Eigengesetzlichkeit gesellschaftlicher Kräfteverhältnisse, wirtschaftlicher Organisationsformen und politischer Institutionen kommt nicht in den Blick. (Dass sich dominierende Haltungen, ein Geist oder Prinzip im Sinne Montesquieus und politische Systeme wechselseitig mitbedingen, wird damit keineswegs geleugnet - wohl aber, dass dies die ganze oder die entscheidende Einsicht sei.)

\section{Unklarheit des politisch-ethischen Argumentationsziels:}

2a. Wichtige Anliegen des Verfassers der Politeia waren, Sokrates und die Philosophie überhaupt durch einen Gegenangriff auf die Polis von Athen zu verteidigen ${ }^{8}$, durch Abgrenzung von den Sophisten, sowie das Anliegen, die spöttische Kritik des Aristophanes durch Angriff auf die Poesie, v.a. in Gestalt des Homer zu kontern'. So betreibt die Politeia einen Mehrfrontenkampf, der zur Verunklärung der praktisch-philosophischen Anliegen führt.

2b. Auch in inhaltlicher Hinsicht hat sich Platon zu viel auf einmal vorgenommen - und zugleich zu wenig - selbst wenn wir uns auf die moralischen Fragen beschränken: Die Fundamentalkritik des als Parade-Sophisten gekennzeichneten Thrasymachos an einem Begriff des Gerechten, der über das Nützliche hinausgeht (Politeia I), dominiert den Diskurs so, dass keine Normen für die Praxis erarbeitet werden: weder für individuell ethisches noch für politisches Handeln gibt Platon konkrete inhaltliche Kriterien ${ }^{10}$ - zu sehr ist er damit beschäftigt, durch Parallelisierung der moralischen Zustände der Seele mit Verfassungsformen (ab 576d), durch die Lehre von den drei hierarchisch zu ordnenden Seelenteilen (493d-440e), durch das Lustargument (583c-586e) und schließlich durch den Mythos von einer postmortalen Belohnung oder Bestrafung (X, 614b-621d) zu erweisen, dass es jenseits des Nützlichen und des Lustvollen ein Gutes und Gerechtes an sich gebe.

3. Das metaphysische Grundproblem zu nennen, könnte trivial erscheinen, ist aber nötig, zumal wegen der Geschlossenheit des platonischen Entwurfs, dem gegenüber eine Kritik der praktischen

\footnotetext{
${ }^{7}$ Vgl. Popper zur geschlossenen Gesellschaft als Organismus (1963, S. 173) und Schmitz 2007, S. 239-242. Den Staat als Organismus zu rekonstruieren, ist sowohl sachlich unangemessen als auch das Organische gründlich missverstanden. Eine Ausnahme bildet nur das pädagogische Prinzip der graduellen, altersgemäßen und ganzheitlichen Erziehung.

${ }^{8}$ Popper (1963, S. 194ff.) unterstellt Platon zudem einen Verrat an Sokrates und Ressentiment gegen die Demokratie, welche mit der Oligarchie im Sinne Spartas auch zwei Onkel Platons beseitigte - was auch nach Schweidler nicht ganz unberechtigt sei (2004, S. 42).

${ }^{9}$ Vgl. Bloom 1991, S. 307ff. und 381f.

${ }^{10}$ Nach Thurnher gehe es Platon immer darum, das gute Ethos der Vorväter durch Wissen krisenfest zu machen (vgl. 2012, S. 331-334). Doch worin besteht es inhaltlich? Und wie wird es im Detail begründet, jenseits seiner die Seele und den Staat erhaltenden Wirkung? „Auch am Ende einer längeren Forschungsreise durch das gewaltige Werk erfährt der Leser nicht, was gerecht und was die Gerechtigkeit ist.“ (Borsche 1996, S. 113)
} 
Konsequenzen alleine ungenügend wirkt: Die Ideenlehre ${ }^{11}$ begründet die Behauptung, dass jenseits der erfahrenen Wirklichkeit, die von Veränderung als Mischung von Sein und Nichtsein gekennzeichnet ist, die ideale, beständige und wahre Wirklichkeit sei ${ }^{12}$. Diese nicht nur ontologische, sondern auch axiologische Zweiweltenlehre strebt unausweichlich von der veränderlichen Welt, damit auch vom Politischen weg. Wenn das Wissen, um sich von bloßer Meinung maximal zu unterscheiden, sich auf unveränderliche Gegenstände beziehen muss, und wenn das Politische als Interagieren von freien Entscheidungen im offenen Raum der Geschichte zwangsläufig von unvorhersehbarer Veränderlichkeit ist, dann ergibt sich zwingend, dass Ideen und Politik sich so fundamental voneinander unterscheiden, dass es zwischen ihnen keine organische Verbindung geben $\mathrm{kann}^{13}$.

So wird schließlich selbst die Erziehung zu einer Dressur im Dienst der Selbsterhaltung des repressiven Staates. Insoweit Veränderung als Ursache allen Übels gesehen wird, erscheinen totalitäre Sozialtechniken (in Analogie zur techne) zur Sicherung des hierarchischen Status quo im Idealstaat als notwendig und gerecht, Freiheit erscheint als Gefahr ${ }^{14}$.

4. Der anthropologische Dualismus bedingt, dass der Mensch eigentlich seine Seele, genauer der höchste „Seelenteil“ Geist ist ${ }^{15}$. Infolgedessen wird der Leib abgewertet. Richtig gesehen ist, dass die Beziehungen zu Ehegatten, Eltern, Kindern, Geschwistern, aber auch zu materiellen Gütern mit der Leiblichkeit des Menschen verbunden sind.

All diese natürlichen Beziehungen sind im Idealstaat zu eliminieren oder abzuschaffen, wenigstens für den Wächterstand (schon Aristoteles war sich nicht klar, inwieweit das für alle Klassen gelten sollte ${ }^{16}$ ). Der resultierende Intellektualismus ist nicht nur eine defiziente

\footnotetext{
${ }^{11}$ Siehe Politeia $\mathrm{V}+$ VI bes. 476a-480a und 507b; von Popper als Essentialismus kritisiert, zusammen mit der historistischen Verfallstheorie Platons (1963, S. 31-34). Diese belegt Thurnher 2012, S. 332 mit weiterführenden Angaben.

${ }^{12}$ Vgl. Phaidon 78d-e. Gerson rekonstruiert die Essenz des Platonismus ohne die Ideenlehre als eine spezifische Form davon, kommt aber gleichwohl zur Schlussfolgerung: „Because there is an all-encompassing hierarchy ordered in terms of complexity and intelligibility, the orientation of investigation is thoroughly ,vertical ${ }^{\text {‘ }}$ and almost never ,horizontal ‘. Thus, there is little room for political philosophy. For political philosophy must start with irreducible political, that is, practical principles. But there cannot be such in Platonism. All principles for Platonism are to be located among that which is relatively simple and intelligible. The concrete and contingent nature of the political militates against the top-down approach." (Gerson 2005)

${ }^{13}$ Vgl. den Mythos von den zwei Weltperioden im Politikos, wonach der Staatsmann die Vergessenheit der ursprünglichen göttlichen Ordnung überwinden müsse durch Erinnerung an deren Prinzipien. Der aprioristische epistemologische Ansatz stößt eben im Bereich des Handlungswissens am schnellsten und deutlichsten an seine Grenzen. Das zeigt sogar der Fortgang des Politikos, hin zu einer aristotelisch anmutenden Bestimmung der normativen Mitte als Maßstab für Handlungen $(284 \mathrm{c}-\mathrm{e})$. Doch sprechen solche späteren Korrekturen wiederum dagegen, das Konzept der Politeia als politische Philosophie (hoch) zu schätzen.

${ }^{14}$ Dies ist der Haupt-Kritikpunkt Poppers. Nach Politikos 293 c-e ist der wahre Staatsmann mit Wissen und Einsicht nicht an eine Zustimmung der Bürger und nicht an bestehende Gesetze gebunden; er müsse nur das Richtige tun, dafür könne er Andersdenkende verbannen oder töten. Tatsächlich ist das nicht das letzte Wort in dem Dialog - nach 301 d-e empfiehlt sich wegen der Missbrauchsmöglichkeiten absoluter Macht bzw. wegen des Misstrauens der Menschen bezüglich der Möglichkeit eines solch göttlichen Herrschers der zweite Weg (300c) in der Spur der idealen Herrschaft (301e) - eine gesetzmäßige Monarchie (302e) als beste aller unleidlichen Regierungsformen. (Nicht nachvollziehbar erscheint deren Charakterisierung als „starre Gesetzesherrschaft“; von Fritz 1968, S. 122-126.) Festzuhalten bleibt aber: Platon kennt keine Möglichkeit, in politischer Beratung das jeweils Beste zu bestimmen, weil keine deliberatio, keine kommunikative Vernunft, sondern nur die Beziehung des einzelnen Weisen zur Erkenntnis, die er wohl im dialektischen Gespräch mit anderen Philosophen gewinnen mag, wozu aber andere Menschen keinen Zugang haben. Selbst wenn das in wissenschaftlichen Fragen richtig wäre, würde das in praktischen Fragen keineswegs automatisch auch gelten, doch eben diese Unterscheidung ist bei Platon signifikant unterentwickelt.

${ }^{15}$ Siehe das homunculus-Bild in Politeia IX 588c-e.

${ }^{16}$ Aristoteles, Politika II,4 1262a 39ff.
} 
Anthropologie, sondern beinhaltet auch totalitäre Ansätze, insoweit angenommen wird, dass die Steuerung des Natürlichen nur mit Gewalt funktioniert ${ }^{17}$.

\section{Individualethische Vagheit:}

5a. Eine Folge des ontologischen, axiologischen und anthropologischen Dualismus ist, dass die zentrale Fähigkeit für das praktische Handeln (individuell und politisch) unwichtig wird: Die Politeia nennt unter den vier Haupt- oder Kardinaltugenden nicht die Klugheit (phronesis) als situationssensible Disposition zum Finden der je besten Handlungsoption im Bereich des Kontingenten ${ }^{18}$, sondern an ihrer Stelle die Weisheit (sophia) ${ }^{19}$ oder Wissenschaft (episteme ${ }^{20}$ als Erkenntnis der Ideen.

5b. Im individualethischen Bereich fällt durch die Abwertung und Abwehr alles Natürlichen auch jeder daraus zu folgernde Maßstab zur Beurteilung konkreter Handlungen weg. Die Vernunft soll im Menschen herrschen über die Triebe, soweit möglich mithilfe der Aggressionskraft, indem sie an der ewigen Idee des Guten Maß nimmt, deren Normierungskraft im Dunkeln von Andeutungen und Postulaten verbleibt. Zugänglich ist sie nach Pol. 506d-e nur der philosophischen Elite, die vielen als esoterische Gruppe mit mündlich tradiertem Geheimwissen erschien. (Folgerichtig wurde diese oberste Idee im Neuplatonismus i.w. spirituell und weniger ethisch gedeutet, womöglich in Ausarbeitung der ungeschriebenen Lehre Platons.)

So finden sich in der Politeia, aber ebenso im Politikos, auch in Bezug auf die ursprüngliche individualethische Frage nach dem Gerechten keine moralischen Kriterien ${ }^{21}$.

\section{Aporie der aporetischen Deutung:}

Die Widernatürlichkeit und Absurdität des von Sokrates propagierten radikalen Kommunismus mag dazu führen, die aporetischen Elemente der Politeia zu betonen, das Scheitern

\footnotetext{
${ }^{17}$ Im Kontext unserer Frage hilft es nichts, daran zu erinnern, dass die Grundaussage sei, dass die Vernunft herrschen solle (etwa Schweidler 2004, S. 38). Diese abstrakte Maxime ist unbestreitbar, aber Praxis beginnt mit der Frage nach dem Wie vernünftiger Ordnung. - K. v. Fritz hebt hervor, dass insbesondere der frühe Platon die individualethische Gewaltlosigkeit gefordert habe, und so will ja auch der Sokrates der Politeia lieber Unrecht erleiden als tun (vgl. Gorgias 508d). Allerdings spricht er durchaus vom „totalitären Aspekt der platonischen Idealstaatskonstruktionen“ (v. Fritz 1968, S. 120) und sieht „Anzeichen dafür, daß unbezweifelbar wirkliche Schüler Platons und Mitglieder der Akademie sowohl zu der Zeit, als Dionys II. noch in Syrakus regierte, als auch nach der Befreiung der Stadt durch Dion für eine rücksichtslosere und skrupellosere Politik eingetreten sind“ (133). Das erweist das Potenzial der Ideologie für die Suspendierung individualethischer Pflichten.

18 Platons Unverständnis für das Misstrauen von Dionys II. ist „eine höchst eindrucksvolle Illustration des Verhältnisses zwischen Philosoph und Politiker, wie der Philosoph überall nur das Moralische sieht, ohne die psychologische Wirkung gewisser Ereignisse zu sehen“ (v. Fritz 1968, S. 70; vgl. 85) - eindeutig ein Mangel an Klugheit.

${ }_{19}$ Politeia 427e-428b. Obwohl Platon 433d phronesis schreibt, in 428b klar als eine Form von episteme erläutert; vgl. Menon $87 \mathrm{c}$.

${ }^{20}$ Auch im Politikos ist die Staatskunst eine Wissenschaft (episteme, 292bff., vgl. 258b), der Wissende steht über dem Gesetz, ohne dass der Inhalt seines normativen Wissens klar würde. Ähnlich in Nomoi: Wenngleich die Verfassungslehre von Nom. VI nicht den Philosophenkönig propagiert, gibt es im Kontrollorgan der „,nächtlichen Versammlung“ (Nom. XII 962d) eine Elite der aufgrund von einem Wissen Herrschenden, das aber selbst im Dunkeln bleibt. Immerhin wird hier der Begriff phronesis verwendet (963c; 964b). Aus dessen gelegentlichem Gebrauch folgt freilich nicht, dass Platon seine spezifische und zentrale Bedeutung erkannt hätte.

${ }^{21}$ Auch Effe, der in Politeia wie Politikos „Wissens-Absolutismus“ konstatiert, stellt fest, „eine detaillierte Erörterung über die Spezifika des staatsmännischen Wissens [...] bleibt mündlicher Esoterik vorbehalten“ (Effe 1996, S. 212).
} 
der leibvergessenen oder leibfeindlichen politischen Utopie ${ }^{22}$, oder die Überlegenheit der Philosophie auch auf dem Gebiet der Komödie im Gegenangriff auf Aristophanes ${ }^{23}$. Auch dieser Interpretationsansatz führt zu dem Ergebnis, dass mit der platonischen Politeia kein Staat zu machen ist.

\section{Reform der Klassengesellschaft?}

7a. Die persönliche Perspektive Platons als eines Adeligen erlaubt ihm, in einer strengen Klassengesellschaft mindestens kein Problem zu sehen. Der Philosoph versucht, durch „Bekehrung“24 der Führungselite die Polis zu reformieren bis hin zur Institution des Philosophenkönigs als Bedingung der Möglichkeit der Reform ${ }^{25}$. Dieses Vorhaben ist schon in der Politeia insoweit stecken geblieben, als die Gesprächspartner des Sokrates, so willig sie diesem auch immer zustimmen, nicht zu höherer Einsicht fähig sind (obwohl Glaucon und Adeimantus als Söhne Aristons immerhin Brüder Platons sind, vgl. 368a).

Aristoteles, der (zeitweise) geduldete Fremdling in Athen, hatte demgegenüber eine andere Perspektive - zwar auch ein gewisses Vorurteil zugunsten der Oberschicht, aber er formulierte ein klares Bekenntnis zur Ausgewogenheit der Kräfte im Staat, wobei die Mittelklasse zur Moderierung entscheidend $\operatorname{sei}^{26}$.

7b. Wäre dementsprechend das Ziel der Politeia nur, die zwei Nachwuchspolitiker Glaucon und Adeimantus auf ihrem (letztlich bescheidenen) Reflexionsniveau zu überzeugen? Das wäre nicht viel mehr als das Niveau des Thrasymachos, als dialektische Abwehr seiner radikalen These: Die Seele hat einen intrinsischen Bezug zur Moralität und leidet selbst unter unrechtem Handeln, das Moralische ist nicht auf das Nützliche reduzierbar. Der praktische Ertrag dieser fundamentalmoralischen Pädagogik ist minimal. Politische Wirkung könnte sie allenfalls indirekt haben in Zusammenhang mit dem elitären Klassenkonzept: Ein paar Söhne der Führungselite sollen von sophistischer Nützlichkeitsmoral und vom Unterworfensein unter ihre Triebe befreit werden.

Das wäre schon etwas mehr als Nichts, aber damit ist kein Staat zu machen.

\section{Literaturverzeichnis}

Primärquellen

Aristotle: Politics, transl. Rackham, Cambridge, MA, Harvard University Press 1932.

Plato: The Republic, transl. Allan Bloom, New York, Harper Collins 1991.

Platonis Opera (Ed. Ioannes Burnet), Oxford, Clarendon 1952-54.

\footnotetext{
22 Bloom 1991, S. 386-389. Vgl. die Diskussionen in der Politeia über die Möglichkeit, den utopischen Idealstaat zu verwirklichen, mit der Tendenz, ihn ,im Himmel als heiliges Musterbild“ (paradeigma) anzusiedeln (X 592b) und die Andeutung einer Möglichkeit zu einer graduellen Annäherung in 472c.

${ }^{23}$ Bloom 1991, S. 381f.

${ }^{24}$ Vgl. die häufige Metapher der „Umkehrung“ oder „Umwendung der Seele“ in Politeia VII.

${ }^{25}$ Siehe die Stellenangaben in Fußnote 3.

${ }^{26}$ Aristoteles: Politika IV, 11 1295a 25-1296b 12.
} 
Sekundärquellen:

Bloom, Allan: Interpretive Essay; in: Plato: The Republic, S. 305-436.

Borsche, Tilman: Die Notwendigkeit der Ideen: Politeia, in: Kobusch/Mojsisch, S. 96-114.

Effe, Bernd: Der Herrschaftsanspruch des Wissenden: Politikos, in: Kobusch/Mojsisch, S. 200-212.

Fritz, Kurt von: Platon in Sizilien und das Problem der Philosophenherrschaft, Berlin (de Gruyter) 1968.

Gerson, Lloyd P.: What is Platonism? Journal of the History of Philosophy 43 (2005), S. 253-276, online: http://individual.utoronto.ca/lpgerson/What_Is_Platonism.pdf [31. Mai 2014]

Kobusch, Theo / Mojsisch, Burkhard (Hrsg.): Platon. Seine Dialoge in der Sicht neuer Forschungen, Darmstadt (Wissenschaftliche Buchgesellschaft) 1996.

Popper, Karl Raimund: The Open Society and its Enemies, vol. 1: The Spell of Plato, New York (Harper \& Row) 1963.

Schmitz, Hermann: Der Weg der europäischen Philosophie. Eine Gewissenserforschung, 1: Antike Philosophie, Freiburg / München (Alber) 2007.

Schweidler, Walter: Der gute Staat. Politische Ethik von Platon bis zur Gegenwart, Stuttgart (Reclam) 2004.

Thurnher, Rainer: Dem Philosophen eine Heimat geben - Zur Sinnbestimmung von Platons Staatsutopie; in: Den Menschen im Blick. Phänomenologische Zugänge, Hrsg. v. Reinhold Esterbauer / Martin Ross, Würzburg (Königshausen \& Neumann) 2012, S. 327-348.

Dr. Markus Riedenauer Professor at Katholische Universität Eichstätt-Ingolstadt Markus.riedenauer@ku.de 